Kennesaw State University

DigitalCommons@Kennesaw State University

Faculty Publications

$12-2001$

\title{
A New System of Parallel Isolated Nonthermal Filaments near the Galactic Center: Evidence for a Local Magnetic Field Gradient
}

Ted N. La Rosa

Kennesaw State University, tlarosa1@kennesaw.edu

Joseph W. Lazio

Naval Research Laboratory

Nasir E. Kassim

Naval Research Laboratory

Follow this and additional works at: https://digitalcommons.kennesaw.edu/facpubs

Part of the External Galaxies Commons, and the Stars, Interstellar Medium and the Galaxy Commons

\section{Recommended Citation}

LaRosa et al TN. 2001. A new system of parallel isolated nonthermal filaments near the galactic center: Evidence for a local magnetic field gradient. Astrophys J 563(1):163.

This Article is brought to you for free and open access by DigitalCommons@Kennesaw State University. It has been accepted for inclusion in Faculty Publications by an authorized administrator of DigitalCommons@Kennesaw State University. For more information, please contact 
The Astrophysical Journal, 563:163-171, 2001 December 10

(C) 2001. The American Astronomical Society. All rights reserved. Printed in U.S.A.

\title{
A NEW SYSTEM OF PARALLEL ISOLATED NONTHERMAL FILAMENTS NEAR THE GALACTIC CENTER: EVIDENCE FOR A LOCAL MAGNETIC FIELD GRADIENT
}

\author{
T. N. LARosa ${ }^{1}$ \\ Department of Biological and Physical Sciences, Kennesaw State University, 1000 Chastain Road, Kennesaw, GA 30144; ted@avatar.kennesaw.edu \\ AND \\ T. Joseph W. LAZIO AND NAMIR E. KASSIM \\ Code 7213, Remote Sensing Division, Naval Research Laboratory, Washington, DC 20375-5351; lazio@rsd.nrl.navy.mil, kassim@rsd.nrl.navy.mil \\ Received 2001 June 1; accepted 2001 August 14
}

\begin{abstract}
We report the discovery of a system of isolated nonthermal filaments approximately 0.5 northwest (75 pc in projection) of Sgr A. Unlike other isolated nonthermal filaments which show subfilamentation, braiding of subfilaments, and flaring at their ends, these filaments are simple linear structures and more closely resemble the parallel bundled filaments in the Galactic center radio arc. However, the most unusual feature of these filaments is that the $20 / 90 \mathrm{~cm}$ spectral index uniformly decreases as a function of length, in contrast to all other nonthermal filaments in the Galactic center. This spectral gradient may not be due to simple particle aging but could be explained by a curved electron energy spectrum embedded in a diverging magnetic field. If so, the scale of the magnetic gradient is not consistent with a large scale magnetic field centered on Sgr A* suggesting that this filament system is tracing a local magnetic field.
\end{abstract}

Subject headings: Galaxy: nucleus - radio continuum: general

\section{INTRODUCTION}

The isolated nonthermal filaments (NTFs) observed in the Galactic center (GC) are unique to that region. They are characterized by extreme length to width ratios (from 10 to $>100)$, highly polarized emission $(30 \%-70 \%)$, strong magnetic fields $(\sim 1 \mathrm{mG})$ aligned along their long axis, and nonthermal spectra (e.g., the $20 / 90 \mathrm{~cm}$ spectral indices, $S \propto v^{\alpha}$, range from $\alpha=-0.4$ to -0.6 , typically steepening above $5 \mathrm{GHz}$ to $\alpha \sim-1.5$ ), for a review see Morris \& Serabyn (1996). Subsequent work has revealed that many isolated NTFs consist of subfilaments braided around each other (e.g., Yusef-Zadeh, Wardle, \& Parastaran 1997; Lang et al. 1999a) and that the surface brightness appears to be a maximum at the intersection of the subfilaments (LaRosa et al. 2000). Lastly, Lang, Morris, \& Echevarria (1999b) and LaRosa et al. (2000) found that the spectral indices in several well studied filaments (the southern and northern threads and the Sgr C filament) are constant with length. The exception is the Snake filament which exhibits a spectral gradient in the region surrounding its major "kink" (Gray et al. 1995).

The relationship between the NTF phenomenon and the parallel bundled filaments in the GC Radio Arc is not clear. The filaments in the Radio Arc show little substructure, have nearly flat spectra and can be observed at frequencies as high as $150 \mathrm{GHz}$ (Reich, Sofue, \& Matsuo 2000). Several theoretical models suggest that the Arc filaments and the NTFs are tracing a large-scale magnetic field that pervades the GC region (e.g., Serabyn \& Morris 1994). At present, there is no consensus interpretation of these structures and new models are under development (e.g., Shore \& LaRosa 1999; Bicknell \& Li 2001; Dahlburg et al. 2001).

A recent wide-field image of the $\mathrm{GC}$ region at $90 \mathrm{~cm}$ (LaRosa et al. 2000) revealed several extended sources well away from the GC itself. One of these, G358.85+0.47,

\footnotetext{
${ }^{1}$ Navy-ASEE Summer Faculty Fellow, Naval Research Laboratory.
}

which is $225 \mathrm{pc}$ in projection from Sgr $\mathrm{A}^{*}$, was found to be the first NTF that is parallel to the Galactic plane (Lang et al. 1999a). This paper reports a detailed study of G359.85+ 0.39, another source discovered on the wide-field image. It is a system of three parallel NTFs located approximately $0.5(\approx 75 \mathrm{pc}$ in projection) northwest of Sgr A. In $\S 2$ we present 90,20 , and $6 \mathrm{~cm}$ observations of this source, made with the Very Large Array (VLA) of the National Radio Astronomy Observatory. ${ }^{2}$ In $\S 3$ we describe alternate scenarios to explain the morphology and spectral index gradient of the source; we present our conclusions in $\S 4$.

\section{OBSERVATIONS AND ANALYSIS}

\subsection{Observations}

The extended source G359.85 + 0.39 was discovered originally on a wide-field, $90 \mathrm{~cm}$ image of the GC region (LaRosa et al. 2000). Figure 1 is a subimage of the original wide-field image and shows this source to consist of a linear structure approximately $8^{\prime}$ long that curves into a semicircular shape with a radius of $2^{\prime}$. The surface brightness over the linear region rises uniformly from about $20 \mathrm{mJy} \mathrm{beam}^{-1}$ at the southeastern end to a peak of $49 \mathrm{mJy}^{-1}$ beam $^{-1}$ near the center before merging into the circular part in the northwest. The surface brightness in the circular region is somewhat patchy and ranges from 15 to $25 \mathrm{mJy}$ beam $^{-1}$. The rms noise on this image is about $5 \mathrm{mJy}^{-1}$ beam $^{-1}$ so we regard both the linear and semicircular structures to be significant. At the distance of the GC $(8.5 \mathrm{kpc})$ the $90 \mathrm{~cm}$ length of $8^{\prime}$ corresponds to a physical length of about $20 \mathrm{pc}$. In order to determine the spectrum and search for substructure in the source, we obtained additional, higher frequency observations.

We observed the source at 6 and $20 \mathrm{~cm}$ with the VLA; Table 1 summarizes the observations. The $20 \mathrm{~cm}$ obser-

\footnotetext{
2 The National Radio Astronomy Observatory is a facility of the National Science Foundation, operated under a cooperative agreement with the Associated Universities, Inc.
} 


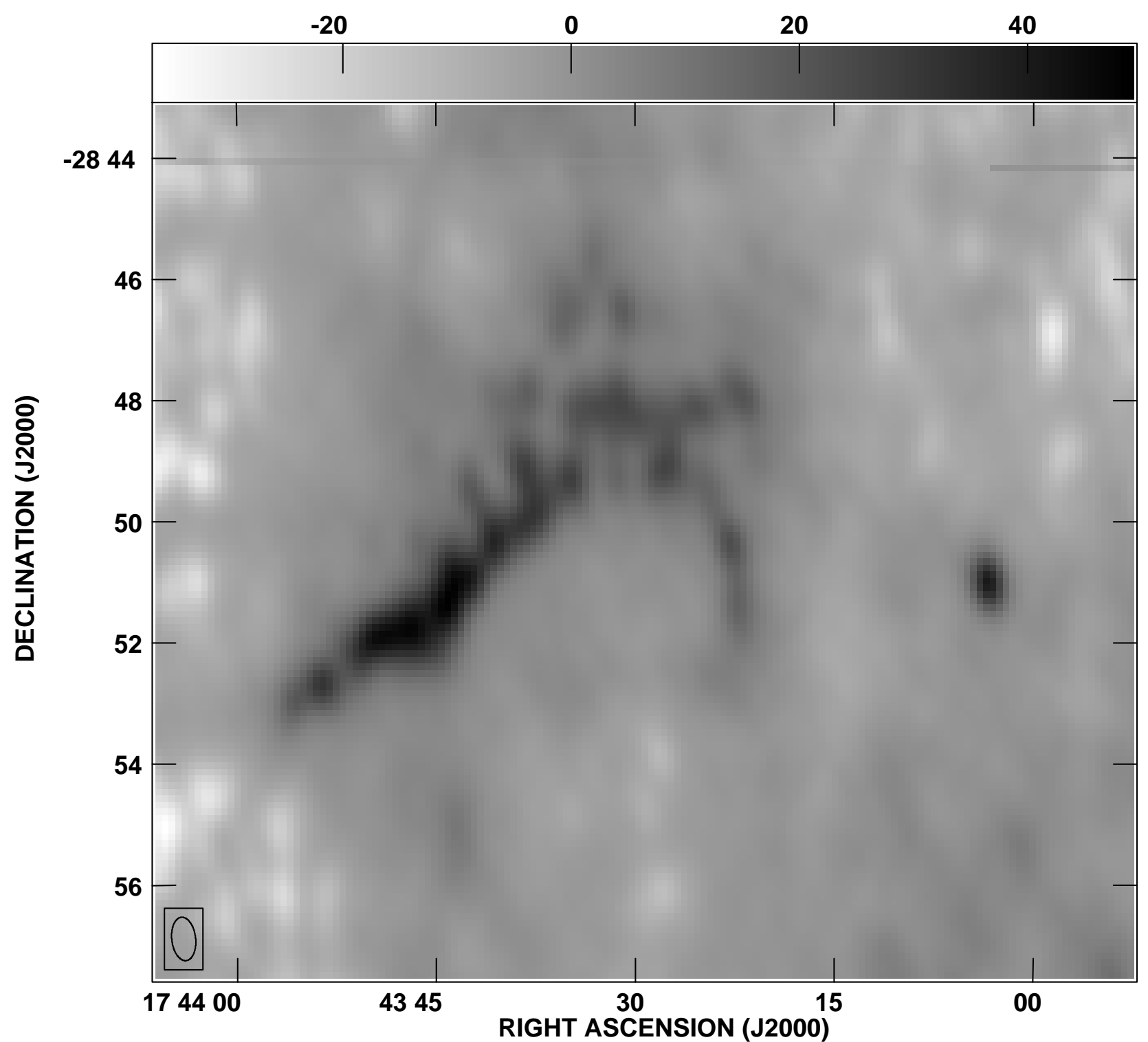

FIG. 1.-Gray-scale representation of G359.85+0.39 at $90 \mathrm{~cm}$ (LaRosa et al. 2000). The resolution is $43^{\prime \prime} \times 23^{\prime \prime}$, the rms noise level is $5 \mathrm{mJy}$ beam ${ }^{-1}$, and the gray scale is linear.

vations were made in 1999 February with the VLA in the DnC configuration. This configuration provides a resolution comparable to that of the $90 \mathrm{~cm}$ observations. Standard processing techniques were used to calibrate and image the visibility data. Figure 2 shows the $20 \mathrm{~cm}$ DnC array image of the region centered on G359.85+0.39. This image shows both the northern and southern threads, as well as the NTFs G359.79+0.17 and G359.54+0.18. At this resolution $\mathrm{G} 359.85+0.39$ appears to be another isolated
NTF with a similar orientation as the other NTFs. Figure 3 shows a subimage centered on G359.85+0.39. The $20 \mathrm{~cm}$ surface brightness is similar to the $90 \mathrm{~cm}$ brightness but peaks at a different location. The source is also approximately $50^{\prime \prime}$ shorter than at $90 \mathrm{~cm}$, and the semicircular emission is not detected.

The semicircular structure visible at $90 \mathrm{~cm}$ (Fig. 1) is notably absent at $20 \mathrm{~cm}$ (Fig. 2). Given the confused nature of the GC, we cannot rule out conclusively the possibility

TABLE 1

VLA OBSERVING LOG

\begin{tabular}{cccccc}
\hline \hline $\begin{array}{c}\text { Wavelength } \\
(\mathrm{cm})\end{array}$ & Epoch & $\begin{array}{c}\text { VLA } \\
\text { Configuration }\end{array}$ & $\begin{array}{c}\text { Bandwidth } \\
(\mathrm{MHz})\end{array}$ & $\begin{array}{c}\text { Integration } \\
\text { Time } \\
(\mathrm{hr})\end{array}$ & $\begin{array}{c}\text { Synthesized } \\
\text { Beam } \\
(\operatorname{arcsec})\end{array}$ \\
\hline $6 \ldots \ldots \ldots \ldots$ & 2000 July 6 & DnC & 50 & 1.5 & $13 \times 9.5$ \\
$20 \ldots \ldots \ldots \ldots$ & 1999 February 27 & DnC & 50 & 2.9 & $43 \times 38$ \\
\hline
\end{tabular}

NOTES.-We summarize only the new observations. The $90 \mathrm{~cm}$ observations are described in LaRosa et al. 2000 . 


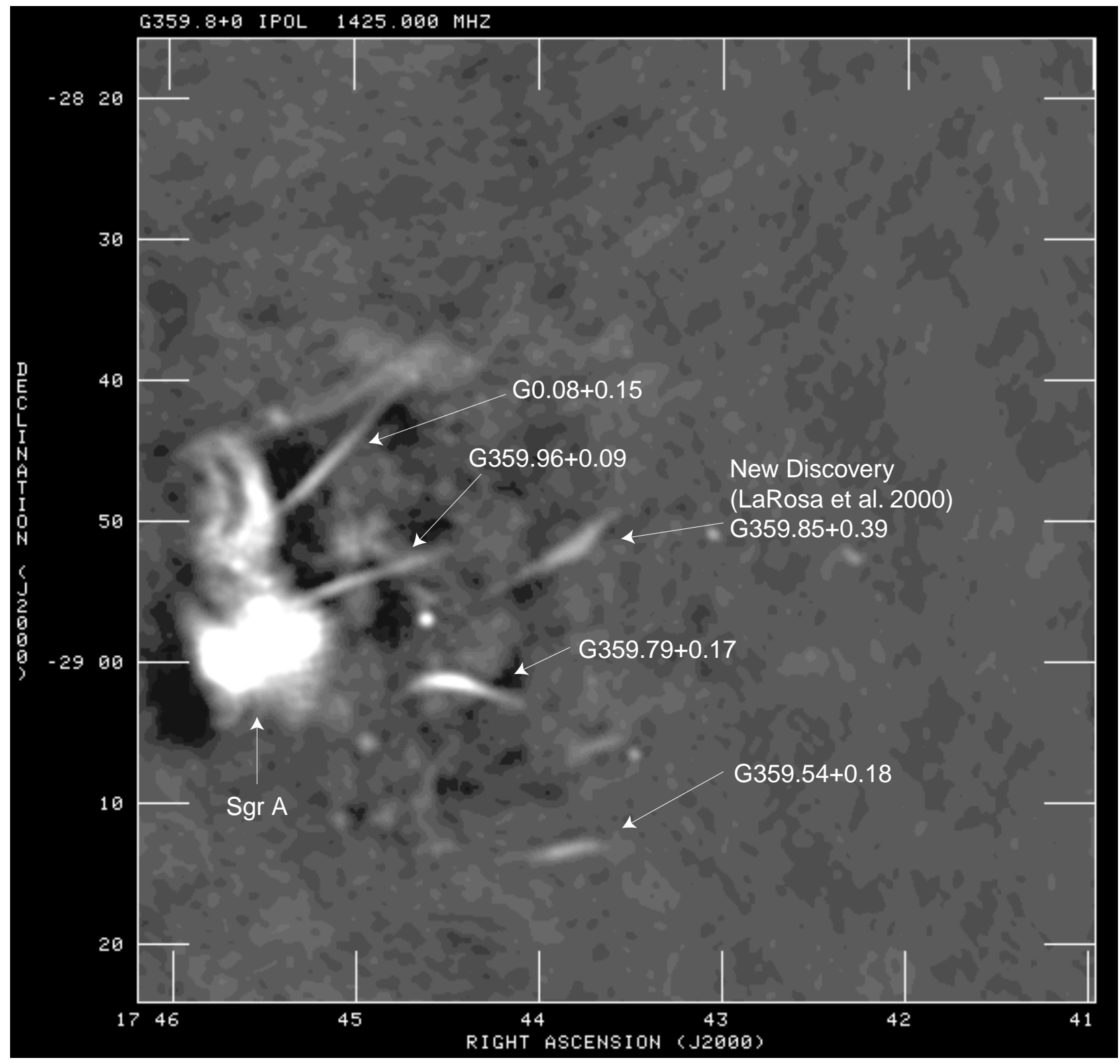

FIG. 2. - Gray-scale representation of the region surrounding G359.85+0.39 at $20 \mathrm{~cm}$. The resolution is 42 ".3 $\times 38.5$, the rms noise level is $2 \mathrm{mJy}$ beam $^{-1}$, and the gray scale is linear.

that this semicircular emission is the superposition of another source, but we regard it as unlikely. First, its morphology is unlike that of most extragalactic sources. While its morphology is similar to that of a (portion of a) supernova remnant, its spectrum is too steep. Our $20 \mathrm{~cm}$ noise level is approximately $1 \mathrm{mJy}$ beam $^{-1}$, so a $3 \sigma$ upper limit on its flux density implies a $20 / 90 \mathrm{~cm}$ spectral index steeper than -1.1 . Second, the surface brightness from the linear part merges smoothly with that of the semicircular part at $90 \mathrm{~cm}$. Finally, the fact that the source is longer at $90 \mathrm{~cm}$ and that the emission peaks at different locations at 20 and $90 \mathrm{~cm}$ suggests a spectral index gradient. Below we show that this source does indeed have a spectral index gradient (from north to south) and the spectral index at the north end of the object is -1.1 , consistent with the spectral index of the semicircular part being steeper than -1.1 .

In 2000 July, $6 \mathrm{~cm}$ continuum observations were made in the VLA DnC configuration. These observations were made in dual polarization mode at 4515 and $4765 \mathrm{MHz}$.
Standard calibration and imaging techniques were used to process the visibility data. Because both the 6 and $20 \mathrm{~cm}$ observations were obtained in the DnC configuration, the $6 \mathrm{~cm}$ image has higher angular resolution.

Figure 4 shows the resulting image. The increased resolution reveals that the linear structure consists of three parallel filaments. As with the 20 and $90 \mathrm{~cm}$ emission the surface brightness in all three filaments rises uniformly from the southeastern end, peaks near the mid point, and declines toward the northwestern end. However, the ultrathin, top filament, although detectable to the eye, is at best only a $3 \sigma$ detection at some points along its length.

Figure 5 shows the linear polarization image. This image indicates that filaments are strongly polarized but also shows considerable polarized emission that does not correspond to any features in total intensity. A similar phenomenon has been found at other wavelengths and in other directions (Gray et al. 1998; Haverkorn, Katgert, \& de Bruyn 2000; Gaensler et al. 2000) and can be explained 

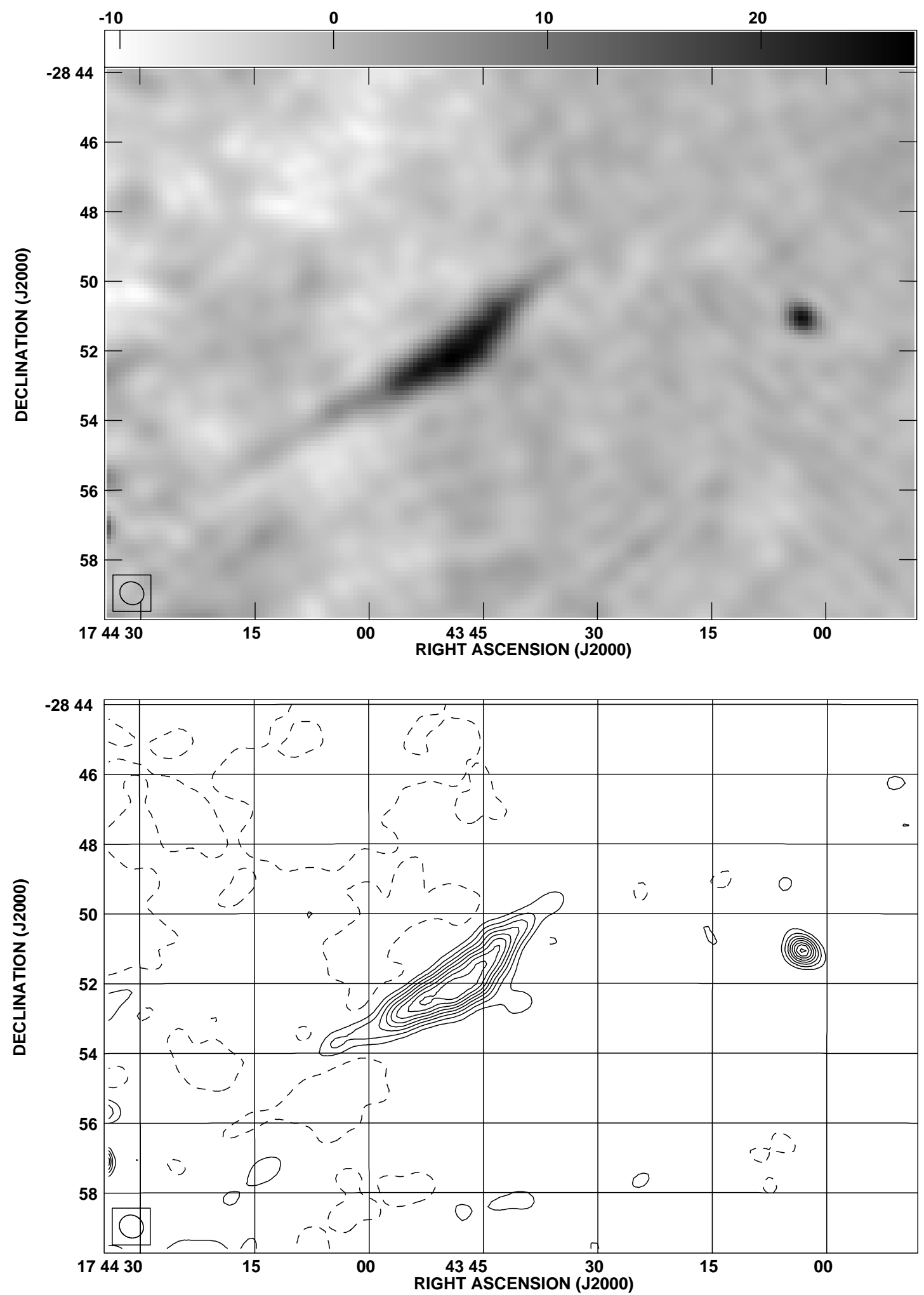

Fig. 3. -G359.85+0.39 at $20 \mathrm{~cm}$. The resolution is $42.3 \times 38.5$, and the rms noise level is $2 \mathrm{mJy}$ beam $^{-1}$. Top: The gray scale is linear. Bottom: The contour levels are 1 mJy beam ${ }^{-1} \times-3,3,6,9, \ldots$.

by a foreground Faraday screen inducing polarization in a diffuse background emission. Analysis of the polarization is beyond the scope of this work and will be discussed elsewhere.

We stress that the subfilaments are parallel since that term has been used to describe many isolated NTFs. However, in all other isolated NTF systems the filaments appear to overlap and cross (Yusef-Zadeh et al. 1997; Lang et al. 1999b). Several also show flaring at their ends. Even at this fairly high resolution no substructure is detectable. Thus, from a morphological perspective these filaments more closely resemble the bundled NTFs in the Galactic center Radio Arc (Yusef-Zadeh, Morris, \& Chance 1984) than the do the isolated NTFs. 

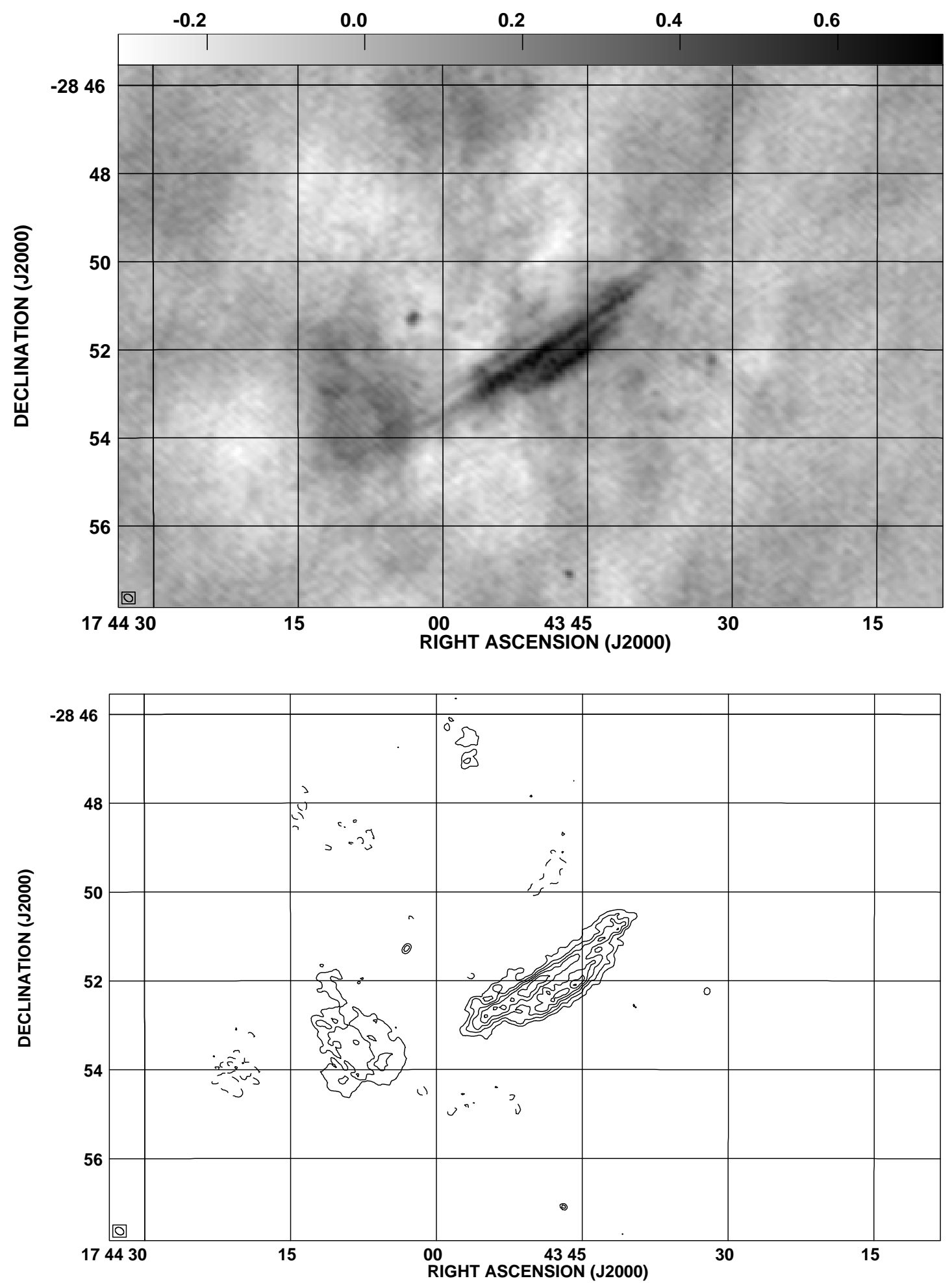

FIG. 4. - G359.85+0.39 at $6 \mathrm{~cm}$. The resolution is $12.5 \times 99^{\prime \prime} 3$, and the rms noise level is $0.08 \mathrm{mJy}$ beam $^{-1}$. Top: The gray scale is linear between -0.31 and $0.73 \mathrm{mJy}$ beam ${ }^{-1}$. Bottom: The contour levels are $0.1 \mathrm{mJy}^{\text {beam }}{ }^{-1} \times-0.225,0.225,0.325,0.425, \ldots$.

To summarize we have observed polarized nonthermal emission from several linear structures with aspect ratios exceeding 10 . These characteristics indicate that these filaments should be classified as GC NTFs.

\subsection{The Spectral Index and Its Gradient}

The spectral index of G359.85+0.39 must change along its length because the peak in emission occurs at different locations at the different wavelengths and because it is longer at $90 \mathrm{~cm}$ than at 6 or $20 \mathrm{~cm}$. Figure 6 compares the 6 and $90 \mathrm{~cm}$ emission.

The similar image resolutions at 90 and $20 \mathrm{~cm}$ make it is possible to estimate the $20 / 90 \mathrm{~cm}$ spectral index with only a minimal convolution. After convolving the $90 \mathrm{~cm}$ image to the $20 \mathrm{~cm}$ resolution, we made cross-cuts perpendicular to the linear part and determined the peak fluxes by fitting a two-point linear baseline at each cross-cut. The length of the cross-cuts was over $300^{\prime \prime}$ on each side of the filament. In 


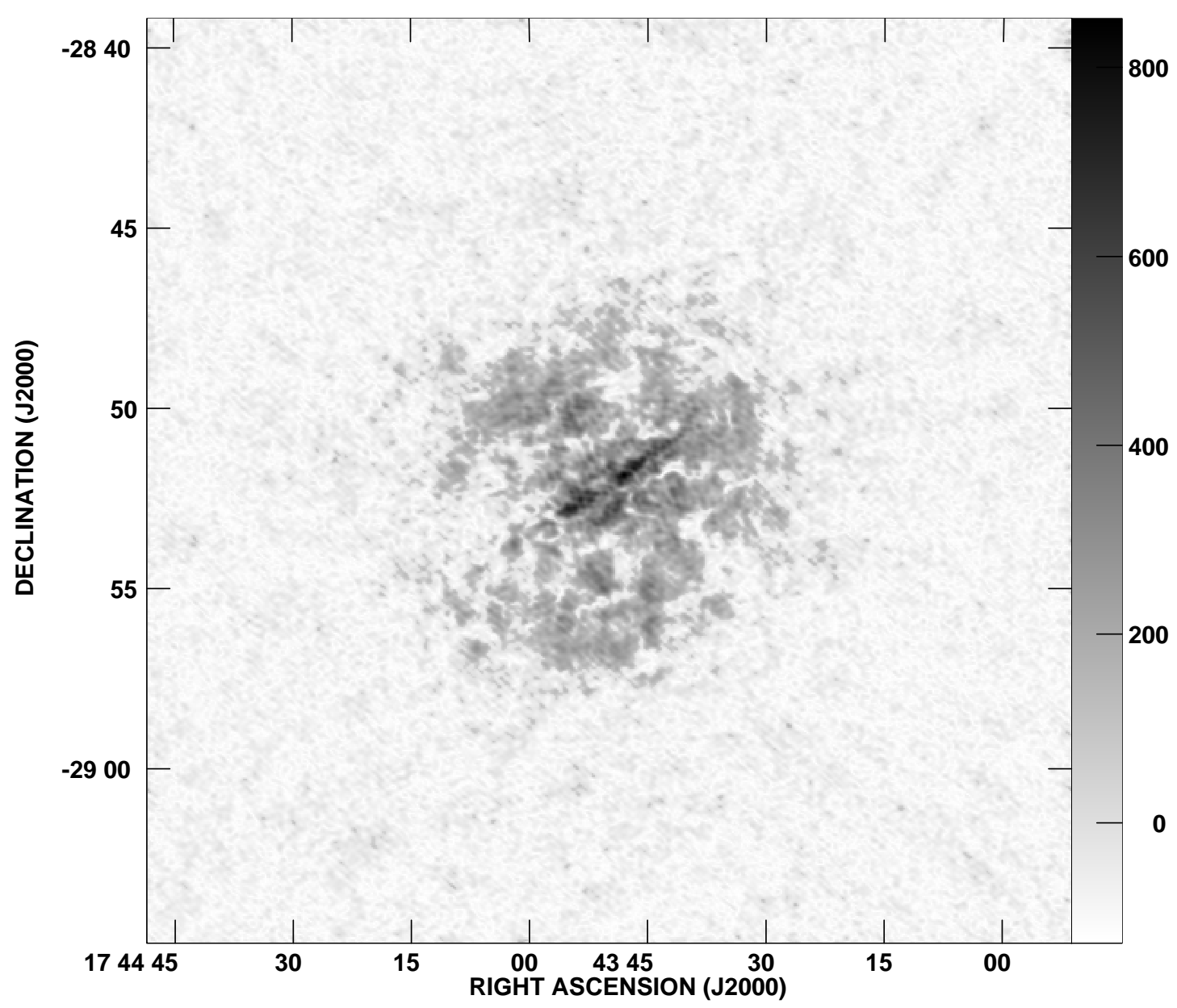

Fig. 5.-Total linear polarization of G359.85+0.39 at $6 \mathrm{~cm}$. The resolution and noise level are similar to that of Fig. 4 . The polarized emission from G359.85 +0.39 is evident at the center of the image, but there is considerable polarized flux that has no correspondence in total intensity (see $\S 2.1$ ).

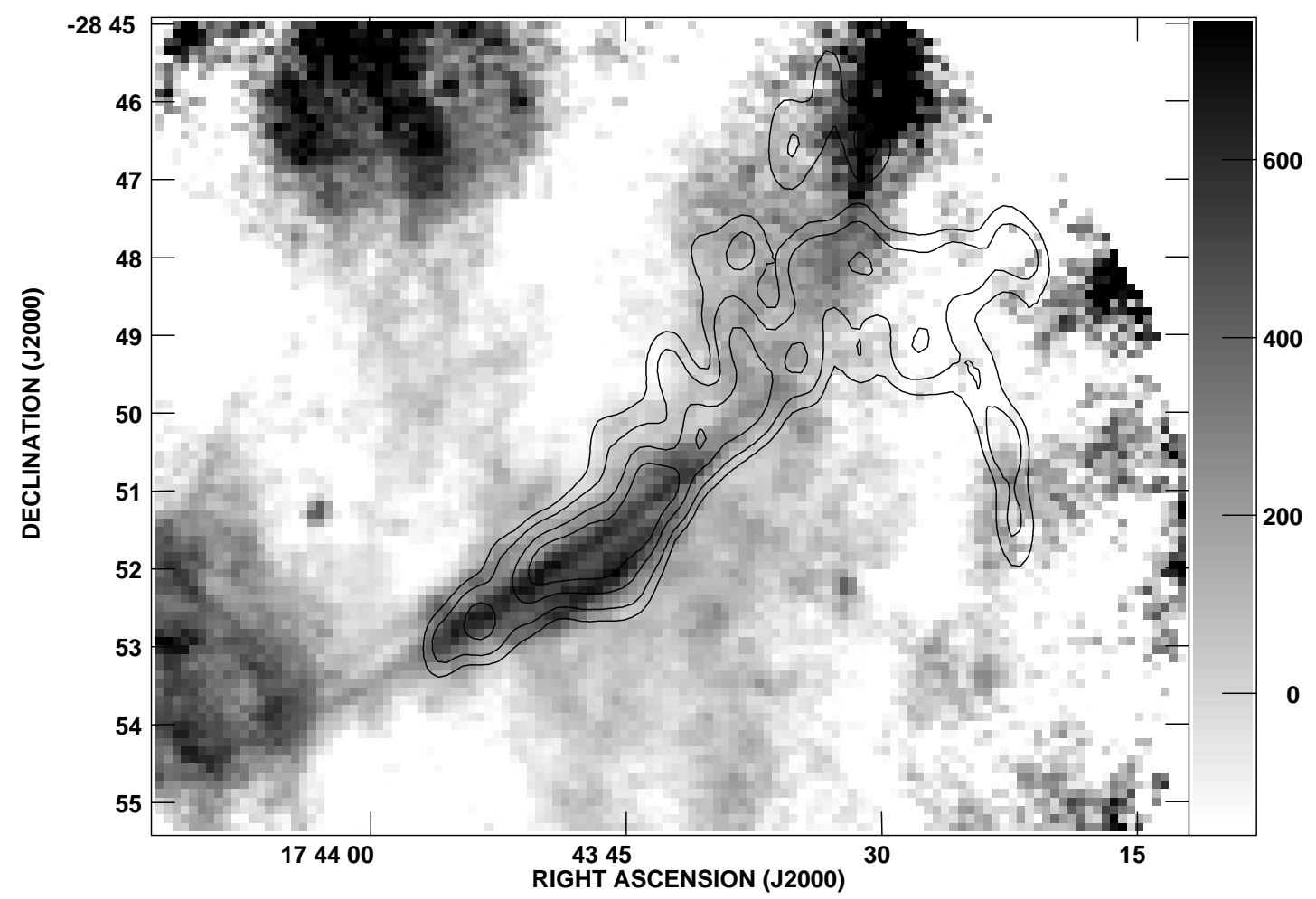

Fig. 6. - Gray-scale of $6 \mathrm{~cm}$ emission (Fig. 4) overlaid on contours of the $90 \mathrm{~cm}$ emission (Fig. 1) 
almost all cross-cuts the endpoints were used to establish the baseline. However, it was also possible to choose reasonable baselines using other points. This leads to a range of possible values for the peak flux at each cross-cut and hence a range in spectral index at each position.

Figure 7 shows the $20 / 90 \mathrm{~cm}$ spectral index plotted as a function of length along G359.85+0.39 with the uncertainties on the points reflecting the uncertainties in the baselines. The spectral index varies smoothly from $\alpha=-0.15$ to -1.1 from south to north, i.e., away from the GC.

There are two possible sources of systematic error in our determination of the spectral index. However, one source of systematic error serves to flatten the derived spectral indices, i.e., the actual spectral index gradient may well be larger than Figure 7 shows. First, the images from which the spectral indices were determined do not have matching spatial frequency coverage. Even though the 20 and $90 \mathrm{~cm}$ images have similar resolutions (see Figs. 1 and 3), the $90 \mathrm{~cm}$ visibility data cover more of the inner $u-v$ plane than do the $20 \mathrm{~cm}$ visibility data. We obtain a rough estimate of the effect of the mismatch of spatial frequency coverage in the following manner: The shortest antenna spacings in the VLA's DnC configuration are approximately $35 \mathrm{~m}$. At $20 \mathrm{~cm}$, these baseline lengths correspond to angular scales of approximately $20^{\prime}$, meaning that structures on scales of $10^{\prime}-20^{\prime}$ are poorly sampled or missing entirely from Figure 2 (and Fig. 3). These angular scales are comparable to or larger than the length of G359.85+0.39 at $20 \mathrm{~cm}$, so we can approximate the effect of the missing short baselines as a constant offset to the brightness of G359.85+0.39. Thus, the spectral indices shown in Figure 7 may be too steep, but, if so, the systematic bias should apply approximately equally to all.

The second possible systematic effect also occurs because of missing short spacings, in particular the "zero spacing," and is evident as the large negative regions surrounding Sgr A in Figure 2. Because Sgr A is so bright, it produces a large "negative bowl" surrounding it (for an additional illustration see Bajaja \& van Albada 1979). This negative bowl may extend well past $\mathrm{G} 359.85+0.39$, but its gradient is directed toward Sgr A. That is, the largest negative regions occur near Sgr A, and their magnitudes become progressively smaller farther away from Sgr A. Thus, this

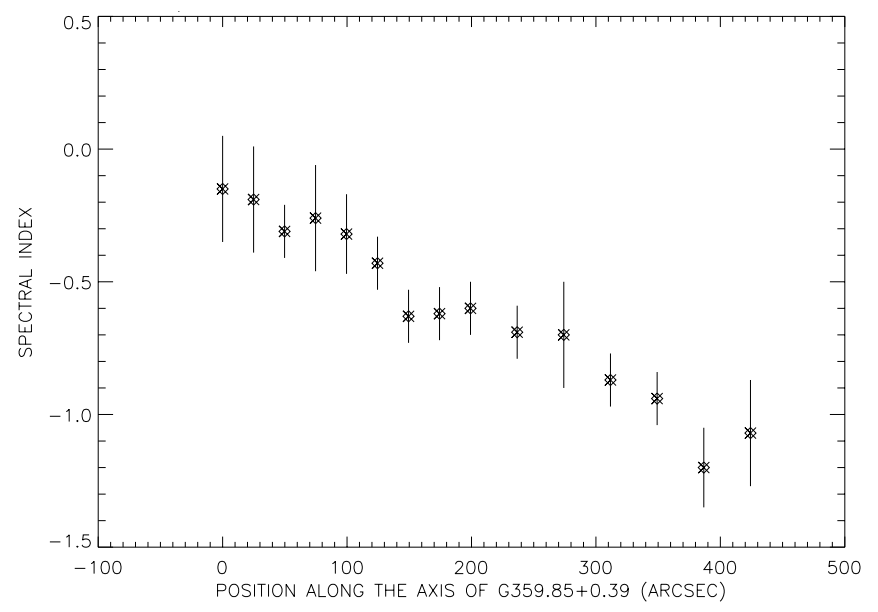

FIG. 7.-The $20 / 90 \mathrm{~cm}$ spectral index as a function of length along $\mathrm{G} 359.85+0.39$. The origin is at the southern end of the filament. Error bars were determine from baseline uncertainties in the individual cross-cuts. negative bowl would tend to depress the brightness at the south end of $\mathrm{G} 359.85+0.39$, where the spectral index is flattest, more so than at the north end. We conclude that the $20 / 90 \mathrm{~cm}$ spectral index gradient shown in Figure 7 is robust and may actually be steeper than shown. Furthermore, there is no evidence of a negative bowl in the region of the semicircular emission. Recall that the semicircular emission would not be detectable if the spectral index was steeper than -1.1 . Given the spectral index is -1.1 in the linear structure and assuming the gradient continues we would not expect to detect the semicircular emission.

We also attempted to use the same method to determine the $6 / 20 \mathrm{~cm}$ spectral index along the length of G359.85+0.39. The differing spatial frequency coverage of the 6 and $20 \mathrm{~cm}$ observations is far more problematic in this case. The missing short spacings mean that angular scales larger than about $25 \%-35 \%$ of the filament's length are poorly sampled or missing. Furthermore, the wide difference in resolution requires a large convolution. We find that the $6 / 20 \mathrm{~cm}$ spectral index is steeper than the $20 / 90 \mathrm{~cm}$ spectral index, with $\alpha$ ranging from -0.9 to -1.3 . This result must be regarded as an upper limit to the spectral curvature. We also found no systematic variation with position. However, given the caveats mentioned above we cannot attach much significance to this result. Additional observations are required to make definitive statements about the $6 / 20 \mathrm{~cm}$ spectral index.

\section{A SPECTRAL INDEX GRADIENT: DISCUSSION}

Previous studies of several NTFs (Lang et al. 1999b; LaRosa et al. 2000) have found that their spectral indices are constant with length. For example, the Sgr C filament exhibits a constant $20 / 90 \mathrm{~cm}$ spectral index $\alpha \approx-0.5$ over its entire length, about $27 \mathrm{pc}$. One prominent exception is the Snake filament, which shows a spectral index gradient at the location of the major kink (Gray et al. 1995). The index is steep at the kink with $\alpha \approx-0.5$ and flattens to 0 moving away from the kink.

By contrast, G359.85+0.39 exhibits a $20 / 90 \mathrm{~cm}$ spectral index gradient, with the flattest spectral index occurring at the southeastern end of the filament, not at the midpoint of the filament where the flux peaks. One might expect the location of the peak flux to coincide with the acceleration site and the flattest spectral index. Clearly, the electron acceleration in this object is more complicated than this simple scenario.

In general, the coherence of the NTFs over many parsecs in a region with a strong ram pressure from the surrounding molecular gas suggests that the magnetic field in these structures is quite strong (Yusef-Zadeh \& Morris 1987a, 1987b). Equating ram pressure with magnetic pressure these authors estimate that the magnetic field of an NTF is of order $1 \mathrm{mG}$. We adopt this value for G359.85+0.39. The synchrotron emissivity corresponds to a number density of relativistic particles of order $10^{-5} \mathrm{~cm}^{-3}$. The background particle density from observations of X-ray emitting gas in the GC region is of order $1 \mathrm{~cm}^{-3}$ (Koyama et al. 1996). The electrons responsible for the $90 \mathrm{~cm}$ emission have an energy of $0.14 \mathrm{GeV}$ and a synchrotron lifetime of $6 \times 10^{4} \mathrm{yr}$, while the $20 \mathrm{~cm}$ emission is from $0.29 \mathrm{GeV}$ electrons with a lifetime of $2.9 \times 10^{4} \mathrm{yr}$.

As discussed above, although our estimate of the $6 / 20 \mathrm{~cm}$ spectral index suggests spectral curvature between 6 and $20 \mathrm{~cm}$, it is not conclusive since we are missing $6 \mathrm{~cm}$ 
flux. However, curved spectra have been found by Lang et al. (1999b) for both the southern and northern NTFs and for the Snake filament by Gray et al. (1995). We therefore argue that spectral curvature is a general property of NTFs.

Curvature is most commonly interpreted in terms of particle aging. Our spectral index measurements indicate a break in the frequency spectrum between 6 and $20 \mathrm{~cm}$. Assuming an initial power-law spectrum, the time $t$ for synchrotron losses to produce the observed curvature is given by $t=3.3 \times 10^{4} \mathrm{yr} B^{-3 / 2} v_{b}^{-1 / 2}$, where the magnetic field $B$ is in $\mathrm{mG}$ and the break frequency $v_{b}$ is in GHz. Using a magnetic field of $1 \mathrm{mG}$, a break frequency between 6 and $20 \mathrm{~cm}$ corresponds to a source age of order $2 \times 10^{4} \mathrm{yr}$. For comparison in a $1 \mathrm{mG}$ field the synchrotron lifetime of a $0.1 \mathrm{GeV}$ electron emitting at a wavelength of $90 \mathrm{~cm}$ is of order $6 \times 10^{4} \mathrm{yr}$. Although we cannot discount the possibility the acceleration mechanism itself generated a curved spectrum and that this is a young source, we will, for purposes of illustration, assume an age of a few tens of thousands of years.

The origin and structure of the NTF magnetic fields is still a matter of debate (e.g., Shore \& LaRosa 1999). The central question is whether the filaments are tracing a largescale, globally ordered magnetic field or are independent local magnetic structures. If part of an ordered large-scale field, we would expect the NTFs are static equilibrium magnetic structures. Alternatively, if they are local, they are more likely evolving dynamic structures. In addition to the magnetic field, the key questions surrounding the NTF phenomenon are the location of the acceleration region and the subsequent transport electrons. There are no detailed models for the acceleration of electrons in the NTFs, and it is often assumed that electron acceleration occurs in a region considerably smaller than the size of the filament (e.g., Serabyn \& Morris 1994; Rosner \& Bodo 1996). The accelerated electrons subsequently expand along the magnetic field and illuminate the rest of the structure. Particle transport is likely to be very different in static structures than it is in dynamic ones. We begin by showing that this filament cannot be considered to be a static equilibrium structure. We then discuss the scenario we favor in which the filament represents a localized enhancement in the GC magnetic field.

\subsection{Magnetic Field Structure and Particle Transport}

We assume acceleration occurs locally and particles stream along the (static) magnetic field. Such anisotropic velocity distributions are unstable and generate MHD waves which in turn scatter the particles. Such resonant scattering (e.g., Wentzel 1974; Melrose 1982, 1994) impedes electron streaming and results in diffusive motion. Resonant scattering by low-frequency plasma waves produces electron pitch-angle scattering without changing the energy of the electrons. Pitch-angle scattering randomly changes the direction of the electrons resulting in diffusion parallel to the large-scale magnetic field. For strong pitch-angle scattering the electrons change direction rapidly and can be confined to a small region of space (Melrose 1994). The magnitude of the spatial diffusion coefficient, $D_{x x}$ is inversely proportional to the pitch angle diffusion coefficient $D_{\alpha \alpha}$ as

$$
D_{x x}=\frac{1}{6} \frac{V^{2}}{D_{\alpha \alpha}},
$$

where $V$ is the particle velocity. The pitch angle diffusion coefficient for electron scattering by whistler or Alfvén waves (ignoring angular factors) is (Melrose 1982)

$$
D_{\alpha \alpha}=\pi^{2} e^{2} \frac{W\left(k_{r}\right)}{p \epsilon},
$$

where $k_{r}=e B / m_{e} c$ is the resonant wave number, $W\left(k_{r}\right)$ is the wave energy density per unit wave number, $p$ is the particle momentum, and $\epsilon$ the particle energy. Expressing this coefficient in terms of the background magnetic energy density $B^{2} / 8 \pi$ gives

$$
D_{\alpha \alpha} \approx 4\left(\frac{B}{1 \mathrm{mG}}\right)\left(\frac{\epsilon}{1 \mathrm{GeV}}\right)^{-1} \frac{k_{r} W\left(k_{r}\right)}{B^{2} / 8 \pi} .
$$

For diffusive motion the root mean square displacement of a particle is $\left\langle\Delta X^{2}\right\rangle=D_{x x} t$. The parameter that determines the transport is the ratio of the wave energy density to the background magnetic energy density. According to Melrose (1982) the maximum in the wave energy level occurs when the scattering reduces the velocity anisotropy to the threshold anisotropy in one scattering time. For $\left(V_{A} / c\right) \sim 10^{-2}$, $k_{r} W\left(k_{r}\right) /\left(B^{2} / 8 \pi\right)$ is of order $10^{-8}$ (Melrose 1982, eq. [7.76]). We conclude that electrons with an energy of $0.14 \mathrm{GeV}$ diffuse about $13 \mathrm{pc}$ in a magnetic field of $1 \mathrm{mG}$ in $4 \times 10^{4}$ yr. Given that the lengths of G359.85+0.39 and other NTFs are of the same order of magnitude, the age indicated by the spectral curvature is consistent with this length if the acceleration occurs in a region whose length is small compared to the length of the filament. However, such diffusion acting alone does not produce a spectral gradient.

The parallel diffusion coefficient due to scattering by selfgenerated waves depends on the first power of the energy. Since the synchrotron lifetime of an electron depends inversely on the first power of the energy, the diffusive length associated with a given timescale is independent of energy. Spectral steepening could be produced by diffusion that depends on a power of the energy that is less than 1. For $D_{x x} \propto E^{\beta}$ with $\beta<1$, then $\Delta X \propto E^{-(1-\beta) / 2}$. For example, if $\beta=1 / 2$, then $\Delta X \propto E^{-1 / 4}$ resulting in fewer higher energy particles at large distances from the acceleration site. A full mathematical treatment, which is beyond the scope of this paper, is required to establish if diffusion by preexisting plasma turbulence could generate the observed spectral steepening. However, in a static magnetic field configuration there is no compelling physical basis to introduce a spectrum of turbulence. We therefore explore transport in a dynamic configuration.

In a dynamic, evolving magnetic field configuration both plasma and MHD turbulence are expected. Furthermore, the magnetic field in a turbulent system could be tangled with a significant random component. Polarimetric studies of other NTFs (e.g., Yusef-Zadeh et al. 1997; Lang et al. $1999 \mathrm{~b})$ show that on the large scales the magnetic field is aligned along the long axis of the NTF suggesting an ordered structure. However, on scales much smaller than a beam (i.e., subparsec), the field could have a significant random component. Cross-field transport would dominate in this situation. Although classical theory predicts that the ratio of parallel to cross field diffusion is of order $10^{-6}$, observations of cosmic rays indicate a ratio of order $10^{-2}$ (Jokopii 1999). A reduction in our previous diffusion coefficient by this factor reduces the diffusion length to $1 \mathrm{pc}$, 
which would certainly require acceleration to occur along the entire length of the filament. Electrons would clearly age before they could fill the filament, but it is not at all clear the transport and distributed acceleration will conspire to produce a uniform spectral gradient. It seems in either the static or dynamic configuration something more is required to explain a uniform gradient.

\subsection{Magnetic Field Variation}

As an alternate possibility to explain spectral index variation, we consider a combination of a curved particle spectrum and a spatially varying magnetic field (Rudnick, Katz-Stone, \& Anderson 1992). In general particles of energy $E$ will emit synchrotron radiation most intensely at a frequency $v_{\mathrm{ob}}=c B^{2} E$. In a region of lower magnetic field, higher energy particles will emit at a given $v_{\mathrm{ob}}$. If the particle energy spectrum is curved, the result is a gradient in the spectral index. Assuming a smoothly declining energy spectrum between two locations with magnetic field strengths of $B_{1,2}$, the change in spectral index is $\delta \alpha=a_{c} \log \left(B_{1} / B_{2}\right)$, where $a_{c}$ is the curvature of the frequency spectrum (Blundell \& Rawlings 2000). Unfortunately, in our case we have only three measured frequencies, and it is not meaningful to fit a second-order polynomial to the spectrum and quantitatively determine a curvature.

If a magnetic field gradient is responsible for the spectral gradient in this source, the much larger northwestern extant of the source at $90 \mathrm{~cm}$ compared to 6 and $20 \mathrm{~cm}$ suggests a fairly rapid decrease in the magnetic field at the this end of the filaments. Such a rapid divergence is not consistent with a globally ordered magnetic field. Morris $(1994,1996)$ suggests that the magnetic field in the GC region is, to first order, a dipole centered on Sgr $\mathrm{A}^{*}$ with a radius of curvature comparable to the size of the central molecular zone, 100 to $200 \mathrm{pc}$. The ratio $B / \nabla B$ is an estimate for the scale of variation. At a projected distance of $75 \mathrm{pc}$, this implies variation on a scale of $25 \mathrm{pc}$. However, the spectral index changes can be measured on a scale of a few parsecs. The region over which the spectral index was measured is $13 \mathrm{pc}$. Over this region 15 cross-cuts were made, and changes in $\alpha$ occur over a few cross-cuts. If a magnetic field gradient is the cause for the spectral gradient, it must be a local field. We conclude that G359.85+0.39 is not tracing a large-scale magnetic field.

\section{SUMMARY}

We have discovered a system of three parallel, nonthermal filaments about $75 \mathrm{pc}$ in projection from Sgr A. The key observational feature of this system is a uniformly decreasing $20 / 90 \mathrm{~cm}$ spectral index as a function of length. It is also noteworthy that the peak flux occurs about midway along the length of the filament, but the flattest spectral index occurs at the end closest to the Galactic center.

There are several explanations for the observed spectral gradient. These include energy dependent diffusion in a turbulent plasma magnetic field configuration and electron acceleration distributed along the length of the filament. However, independent of the electron acceleration and transport, a simpler and more natural scenario is that the magnetic field is varying along the length of the filament. A spectral gradient is a natural consequence of a curved electron energy spectrum radiating in a decreasing magnetic field. If so, the gradient scale length of a few parsecs is not consistent with a large-scale field centered on Sgr A. This result suggests that this nonthermal filament system is not necessarily tracing a large-scale magnetic field. Observations at additional frequencies could be used to determine the spectral curvature and provide specific constraints on the magnetic field structure.

We thank S. Shore for several stimulating discussions, C. Lang for assistance with the observations and discussions in the early stages of this work, and the referee for several suggestions that improved the presentation of this work. Basic research in radio astronomy at the NRL is supported by the Office of Naval Research. T. N. L. was supported in part by a Master Scholarship grant from Kennesaw State University.
Bajaja, E., \& van Albada, G. D. 1979, A\&A, 75, 251

Bicknell, G., \& Li, J. 2001, ApJ, 548, L69

Blundell, K. M., \& Rawlings, S. 2000, AJ, 119, 1111

Dahlburg, R. B., Einaudi, G., Shore, S. N., \& LaRosa, T. N. 2001, ApJ, submitted

Gaensler, B., Dickey, J., McClure-Griffiths, N., Green, A., Wieringa, M., \& Haynes, R. 2000, ApJ, 549, 959

Gray, A. D., Landecker, T. L., Dewdney, P. E., \& Taylor, A. R. 1998, Nature, 393, 660

Gray, A. D., Nicholls, J., Ekers, R. D., \& Cram, L. E. 1995, ApJ, 448, 164

Haverkorn, M., Katgert, P., \& de Bruyn, A. G. 2000, A\&A, 356, L13

Jokopii, J. R. 1999, in Interstellar Turbulence, ed. J. Franco \& A. Carraminana (Cambridge: Cambridge Univ. Press), 70

Koyama, K., Maeda, Y., Sonobe, T. Takeshima, T. Tanaka, Y., \& Yamauchi, S. 1996, PASJ, 48, 249

Lang, C. C., Anantharamaiah, K. R., Kassim, N. E., Lazio, T. J. W., \& Goss, W. M. 1999a, ApJ, 521, L41

Lang, C. C., Morris, M., \& Echevarria, L. 1999b, ApJ, 526, 727

LaRosa, T. N., Kassim, N. E., Lazio, T. J. W., \& Hyman, S. D. 2000, AJ, 119,207

\section{REFERENCES}

Melrose, D. B. 1982, Plasma Astrophysics (London: Gordon \& Breach) .1994, Plasma Astrophysics, Saas Fee Advanced Course 24 (Berlin: Springer)

Morris, M. 1994, in The Nuclei of Normal Galaxies, ed. R. Genzel \& D. Harris (Dordrecht: Kluwer), 185

. 1996, in IAU Symposium 169, Unsolved Problems of the Milky Way, ed. L. Blitz \& P. Teuben (Dordrecht: Kluwer), 247

Morris, M., \& Serabyn, E. 1996, ARA\&A, 34, 645

Reich, W., Sofue, Y., \& Matsuo, H. 2000, PASJ, 52, 355

Rosner, R., \& Bodo, G. 1996, ApJ, 470, L49

Rudnick, L., Katz-Stone, D., \& Anderson, M. 1992, in Particle Acceleration in Cosmic Plasmas, ed. G. P. Zank, \& T. K. Gaisser (New York: AIP), 424

Serabyn, E., \& Morris, M. 1994, ApJ, 424, L91

Shore, S. N., \& LaRosa, T. N. 1999, ApJ, 521, 587

Wentzel, D. G. 1974, ARA\&A, 12, 71

Yusef-Zadeh, F., \& Morris, M. 1987a, ApJ, 322, 721 $1987 \mathrm{~b}, \mathrm{AJ}, 94,1128$

Yusef-Zadeh, F., Morris, M., \& Chance, D. 1984, Nature, 310, 557

Yusef-Zadeh, F., Wardle, M., \& Parastaran, P. 1997, ApJ, 475, L119 